Peer-Reviewed

Citation: Araj F. et al. (2019)

"Refining Auscultation of Left Ventricular Assist Devices: Insights From

Phonocardiography."

The VAD Journal, 5. doi: https://doi.org/10.13023/VAD.201 $\underline{9.06}$

Editor-in-Chief: Maya Guglin, University of Kentucky

Received: February 10, 2019

Accepted: April 29, 2019

Published: May 2, 2019

() 2019 The Author(s). This is an open access article published under the terms of the Creative Commons Attribution-

NonCommercial 4.0 International License

(https://creativecommons.org/lice nses/by-nc/4.0/), which permits unrestricted non-commercial use, distribution, and reproduction in any medium, provided that the original author(s) and the publication source are credited.

Funding: Not applicable applicable

\title{
Refining Auscultation of Left Ventricular Assist Devices: Insights From Phonocardiography
}

\author{
Faris Araj”, Alpesh Amin, Julie Cox, and Pradeep Mammen \\ University of Texas Southwestern Medical Center \\ *Corresponding author: faris.araj@utsouthwestern.edu
}

Keywords: Left ventricular assist device, auscultation, phonocardiography

There have been over 15,000 continuous flow left ventricular assist devices (LVADs) implanted in the United States ${ }^{1}$. As the care of these patients expands into the general community, it is important for providers at all levels to be familiar with the sound of a normal LVAD. The sound generated is normally described as an "LVAD hum". That non-descriptive term may be misunderstood as all LVADs or "hums" are the same, when in fact the sound produced is unique to each device. Another common misconception held by some providers, is the absence of heart sounds in a normally functioning LVAD. Using apex phonocardiography we were able to better visualize these unique characteristics (Figures 1-4, phonocardiograms of three United States Food and Drug Administration approved durable LVADs), and suggest a refined description of each device sound. The recordings were made on normal functioning LVADs using the EKO Core stethoscope attachment (EKO, Berkeley, CA). 


\begin{tabular}{|l|l|}
\hline Abbreviation & Meaning \\
\hline M & Monotone \\
\hline Blue arrows & Native heart sound \\
\hline Green arrows & Abrupt decrease in speed \\
\hline Red arrows & Abrupt increase in speed \\
\hline CT & Crescendo tone \\
\hline DT & Decrescendo tone \\
\hline CD & Crescendo-decrescendo \\
\hline AP & Artificial pulse \\
\hline
\end{tabular}

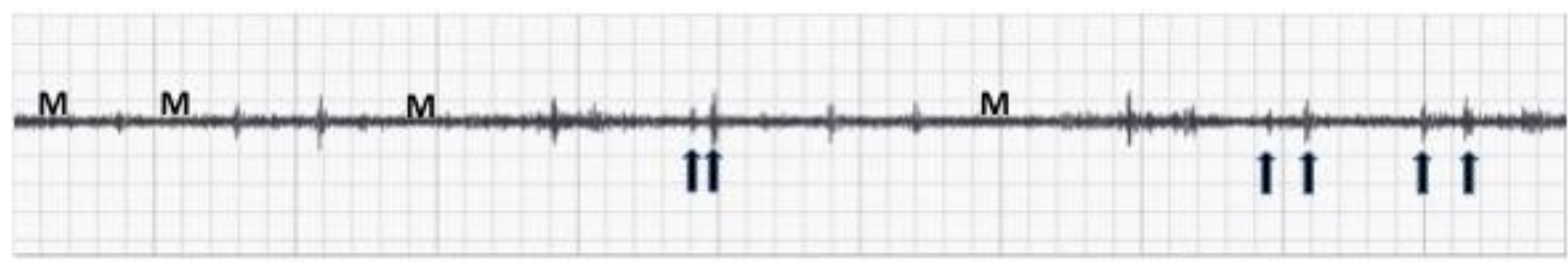

Figure 1: HeartMate II

The HearMate II (Abbott, Inc. Abbott Park, IL) device is a continuous flow LVAD that uses axial flow technology. ${ }^{2}$ Normally the pitch is medium to high in intensity. The phonocardiogram shows a predominantly uniform or monotone $(\mathrm{M})$ sound without fluctuations in sound amplitude, irrespective of native heart sounds (arrows).

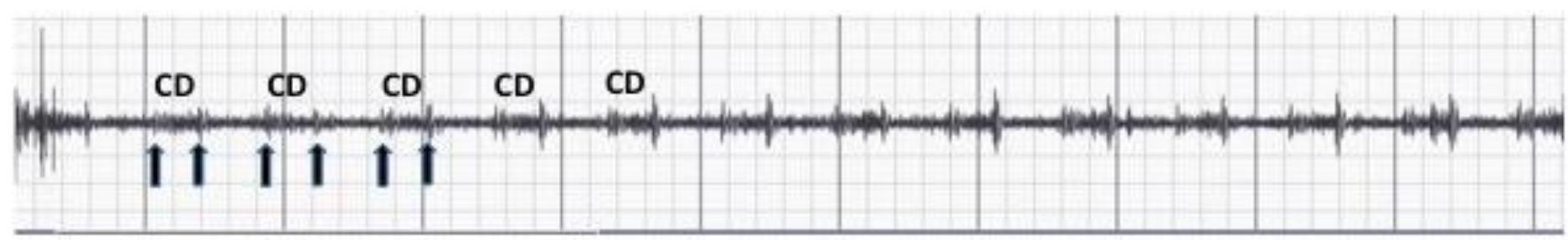

Figure 2: HeartWare- Lavare Cycle off 


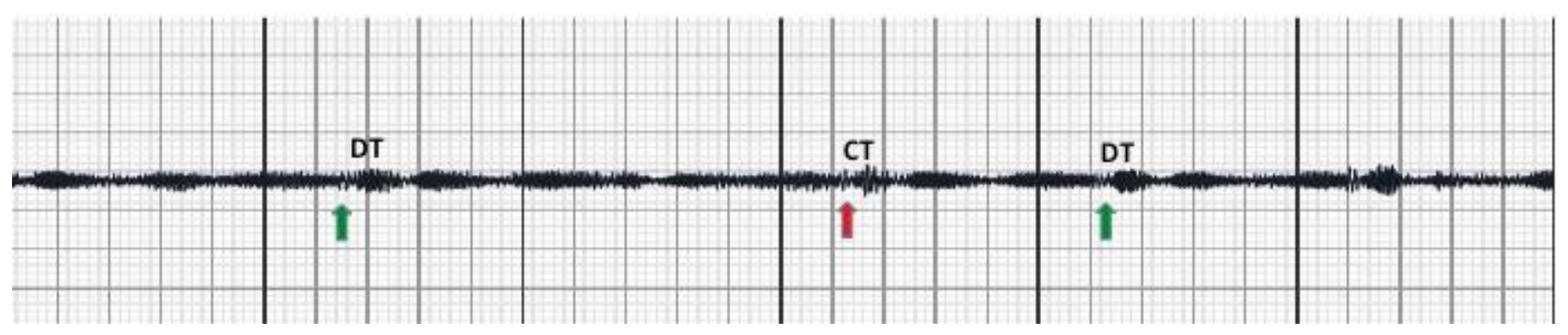

\section{Figure 3: HeartWare- Lavare Cycle on}

The HeartWare (Medtronic, Minneapolis, MN) is a continuous flow LVAD that uses centrifugal flow technology.2 Normally, the pitch is medium to high in intensity. The phonocardiogram shows a baseline monotone sound interspersed with repetitive crescendo-decrescendo (CD) within the confines of the native first and second heart sound (either aortic, pulmonic, or a combination). The sound is louder during systole.

The HeartWare operation can be set to increase washing of the left ventricle and pump, termed the Lavare Cycle. This cycle consists of abrupt speed decrease (arrow and decrescendo tone (DT) for 2 seconds, followed by speed increase (arrow and crescendo tone (CT) for 1 second, followed by the return to baseline speed settings (arrow and decrescendo tone (DT) . The cycle is repeated every 60 seconds.

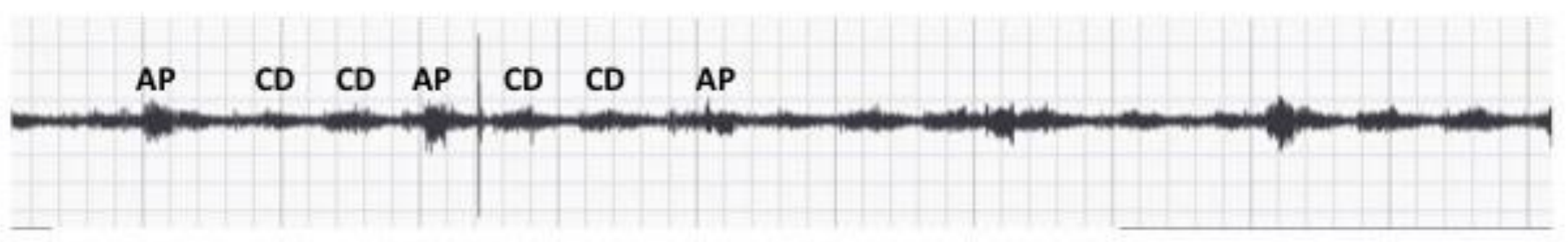

Figure 4: HeartMate III

The HeartMate III (Abbott, Inc. Abbott Park, IL) is a continuous flow LVAD that uses centrifugal technology. ${ }^{3}$ Unique to this device is the presence of an artificial pulse that occurs every $2 \mathrm{sec}$. Normally the pitch is medium in intensity. The phonocardiogram shows a baseline of crescendo decrescendo sound (approximately 2-3 cycles), which is then interrupted by a higher amplitude sound 
with components above and below the baseline. This latter sound is generated by the artificial pulse cycle.

\section{Discussion}

It is well known that the use of cardiac auscultation skills to identify cardiac pathology among trainees is poor, and that the emphasis on this skill set has diminished. ${ }^{4,5}$ Data suggest that incorporating digital stethoscopes in the learning process, can improve trainee auscultatory performance. ${ }^{6,7}$ Since auscultation of the pump is an important part of the physical examination of a patient with a durable LVAD, an audio-visual learning experience (e.g. phonocardiography) may be a better tool for teaching. Additionally, the sounds can be saved and played back to increase familiarity with normal device sound.

It is the authors' belief that a more refined understanding of an LVADs unique sound profile may help clinicians identify changes in sound that may reflect underlying device pathology, immediately at the bedside. Slaughter et al used an aquatic hydrophone system to detect 1 st generation pulsatile LVAD device failure. ${ }^{8}$

More recently Kaufmann et al, and Yost et al used analysis to evaluate to study current generation continuous flow LVADs. The former analyzed the HeartWare in vivo, and the latter the Heartmate II both in vitro and in vivo. The presence of a third harmonic sound was suggestive of pump thrombosis in the HeartWare device, and a reduction of spectral energy over a sound frequency range in the HeartMate II..$^{9,10}$ Although both groups used more complex and sophisticated sound analysis than simple phonocardiography, the concept was simple: a thrombus on the rotor leads to imbalance, and that can lead to a change in device sound.

The main findings from our data:

1. Not all LVAD hums are the same- each device has its own unique sound characteristic.

2. The emitted sound can be recorded using a modern-day stethoscope, or stethoscope attachment.

3. The sound generated can be reviewed and analyzed.

4. To our knowledge no other publication has comprehensively displayed the phonocardiograms of the three main FDA approved durable LVADs. 


\section{References}

1.Kirklin J, Pagani F, Kormos R. Eighth Annual INTERMACS Report: Special focus on framing the impact of adverse events. J Heart Lung Transplant 2017;36: 10801086

2. Mancini D, Colombo P. Left Ventricular Assist Devices: A Rapidly Evolving Alternative to Transplant. JACC 2015; 65: 2542-2555

3. Mehra M, Yoshifumi N, Uriel N et al. A Fully Magnetically Levitated Circulatory Pump for Advanced Heart Failure N Engl J Med 2017; 376:440-450

4. Mangione S, Nieman LZ. Cardiac auscultatory skills of internal medicine and family practice trainees. A comparison of diagnostic proficiency. JAMA 1997; 278 (9): 717-722

5. Mangione S, Nieman LZ, Gracely E, Kaye D. The teaching and practice of cardiac auscultation during internal medicine and cardiology training. A nationwide survey. Ann Intern Med 1993; 119 (1):47-54

6. Legget ME, Toh M, Meintjes A, Fitzsimmons S, Gamble G, Doughty RN. Digital devices for teaching cardiac auscultation-a randomized pilot study. Med Educ Online 2018; 23 (1) 1-7

7. Mesquita CT, Reis JC, Simoes LS et al. Digital stethoscope as an innovative tool on the teaching of auscultatory skills. Arq Bras Cardiol 2013; 100 (2): 187-189

8. Slaughter MS, Feldman, CM, Sobieski MA et al. Utilization of Acustic Signatures to Identify HeartMAte XVE Device End -of Life. JHLT 2007;26: 579-583

9. Kaufmann F, Hörmandinger C, Stepanenko A et al. Acoustic spectral analysis for determining pump thrombosis in rotary blood pumps. ASAIO J. 2014; SepOct;60(5):502-507.

10. Yost GL, Royston TJ, Bhat G, Tatooles AJ. Acoustic Characterization of Axial Flow Left Ventricular Assist Device Operation In Vitro and In Vivo. ASAIO J. 2016; Jan-Feb;62(1):46-55 